BSG Polyp Surveillance guidelines (originally published in 2002). We chose different time periods to study including 2002, 2005-2006, 2009 (50 patients each) and 2011 (100 patients). We had previously conducted an audit in 2010 (unpublished) and following this embarked on a programme of endoscopist education to improve compliance data.

Results Compliance with the BSG Polyp Surveillance guidelines was $33 \%$ in $2002,65 \%$ in $2005 / 2006,57 \%$ in 2009 and $98 \%$ in 2011. Based on our unit's activity 3832 colonoscopies were performed in 2011 with an overall polyp detection rate of 34.6\% (1325.9 colonoscopies). 98\% compliance with BSG guidelines would have resulted in inappropriate advice being given in 26.5 of those colonoscopies. For every $10 \%$ reduction in compliance against the BSG standard (and for our unit based on 2011 figures) an additional 123.5 polyp positive colonoscopies would receice inappropriate guidance on polyp follow-up. In our experience (unpublished), inappropriate advice results in an increase in frequency of follow-up colonoscopies as endoscopists overestimate the patient's future risk of advanced colorectal neoplasia. This potentially has huge resource and organisation implications.

Conclusion We demonstrate a big improvement in compliance with the BSG polyp surveillance guidelines at our unit following a period of endoscopist education in 2010. With demand for colonoscopy projected to rise $5 \%-10 \%$ year on year and with limited ability to increase capacity under current financial constraints, appropriate patient selection for colonoscopy is essential. Failure to comply with the BSG guidelines has significant financial, organisational and patient implications. We recommend all units validate waiting lists for patients on a polyp surveillance programme.

Competing interests None declared.

\section{PWE-088 NEGATIVE LOWER GI INVESTIGATIONS: MISS RATES IN COLORECTAL CANCER}

doi:10.1136/gutjnl-2012-302514d.88

${ }^{1} \mathrm{~L}$ To, ${ }^{1} \mathrm{~S}$ Mills, ${ }^{1} \mathrm{~T}$ Townsend, ${ }^{2} \mathrm{~K}$ Leiper. ${ }^{1}$ University of Liverpool, Liverpool, UK; ${ }^{2}$ Royal Liverpool University Hospital, Liverpool, UK

Introduction Retrospective studies have confirmed that colonic investigations may miss a diagnosis of colorectal cancer (CRC) with a wide variation in reported miss rates. ${ }^{1} 2$ Colorectal cancer miss rates of up to $12 \%, 22 \%$ and $50 \%$ have been reported for colonoscopy, barium enema and sigmoidoscopy, respectively.

Methods A retrospective study was conducted to determine the diagnostic miss rate of colorectal cancer at our institution. All patients diagnosed with colonic or rectal adenocarcinoma between 2006 and 2010 were identified from the Royal Liverpool and Broadgreen University Hospital Trust histopathology database. Data were collected using the computerised systems and case notes. A miss was defined as a patient investigated with barium enema (BE), CT abdo-colon enhanced (CTACE), CT colonoscopy (CTC), flexible sigmoidoscopy and/or colonoscopy, and discharged without being followed-up or diagnosed with CRC in the 5 years preceding the subsequent CRC diagnosis.

Results During the study period, 579 patients were diagnosed with colorectal cancer. The notes were irretrievable or had insufficient documentation in 5 cases. Twenty-two (3.8\%) cases were considered misses: $5(0.8 \%)$ were administrative misses, where patients were lost to follow-up, or they cancelled or failed to attend an appointment; in one case $(0.2 \%)$, there was a clinician-associated miss, where an inappropriate choice of investigation was performed (a flexible sigmoidoscopy missed a proximal CRC); and 16 (2.8\%) were technical misses, where CRC was missed with an appropriate choice of investigation. Of the technical misses, $10(1.7 \%)$ were radiological $(0.7 \%, 0.7 \%$ and $0.3 \%$ for $\mathrm{BE}, \mathrm{CTACE}$ and CTC respectively) and 8
(1.4\%) were endoscopic ( $0.5 \%$ and $0.9 \%$ for flexible sigmoidoscopy and colonoscopy). $45 \%$ of missed cancers were left-sided and below the splenic flexure.

Conclusion This study has shown a lower miss rate in our institution than previously reported, ${ }^{3}$ and when compared with other studies.

Competing interests None declared.

\section{REFERENCES}

1. Frenette CT, Strum WB. Relative rates of missed diagnosis for colonoscopy, barium enema, and flexible sigmoidoscopy in 379 patients with colorectal cancer. $J$ Gastrointest Cancer 2007:38:148-53.

2. Somasekar A, James L, Stephenson BM, et al. The value of auditing negative lowe $\mathrm{GI}$ investigations preceding a final diagnosis of colorectal cancer. Colorectal Dis 2009;11:740-4.

3. Graham M, et al. Endoscopy 2005;37:A275

\section{PWE-089 THE ROLES OF CYP2C40 AND CYP2C55 IN PREVENTING COLON CANCER}

doi:10.1136/gutjnl-2012-302514d.89

${ }^{1} \mathrm{M}$ Albert, ${ }^{* 2} \mathrm{~A}$ Bennett. ${ }^{1}$ School of Medicine, University of Nottingham, Nottingham, UK; ${ }^{2}$ School of Biomedical Sciences, University of Nottingham, Nottingham, UK

Introduction Certain Cytochrome P450 (CYP)-dependent arachidonic acid (AA) metabolites are thought to induce therapeutic effects in the colon via activation of peroxisome proliferator activated receptors (PPARs), specifically the PPAR $\alpha$ subtype. The activation of PPAR $\alpha$ leads to changes in the expression and activity of target genes and other transcription factors such as COX-2, NF-KB and AP-1, resulting in anti-inflammatory and anti-tumorigenic effects. CYP2C40 and CYP2C55 are two recently discovered CYPs, isolated from the murine intestinal tract. Their metabolites include 16-HETE, 8,9-EET and 14,15-EET which have been shown to have anti-inflammatory effects both in vitro and in vivo. Evidence suggests that CYP2C40 and CYP2C55 may have a potential therapeutic role to play in colon tumorigenesis. This study aims to determine whether PPAR activation leads to an up-regulation in the expression of CYP2C40 and CYP2C55.

Methods CYP2C40 and CYP2C55 promoter regions were isolated from murine DNA via PCR and inserted into a luciferase plasmid (pGL4.10). Plasmid DNA was cloned following transfection into highly competent cells and purified via Midi-Prep recovery. Purified plasmids were transfected into COS-7 and HCA-7 cells and the cells were treated with PPAR $\alpha, \beta$ and $\gamma$ ligands Wy14643, GW0742 and Rosiglitazone (COS-7 cells had PPAR $\alpha / \beta / \gamma$ over-expressed). Cells were harvested after $24 \mathrm{~h}$ incubation and luciferase activity (equivalent to gene expression) was measured in relative light units (RLU) using a reporter assay system.

Results In COS-7 cells PPAR $\alpha, \beta$ and $\gamma$ ligands led to a significant increase in CYP2C40 RLU from a baseline measurement of (mean ( \pm SD)) 235 (20) to 726 (45), 458 (61) and 466 (42) for PPAR $\alpha, \beta$ and $\gamma$ respectively. CYP2C55 showed a significant increase from 154 (6) to 263 (10) and 354 (21) for PPAR $\alpha$ and $\beta$ respectively $(p=0.001)$. HCA-7 cells were shown to only express endogenous PPAR $\alpha$ and following incubation with PPAR $\alpha, \beta$ and $\gamma$ ligands a significant RLU increase was observed in CYP2C40 from 55 (13) to 126 (17) and in CYP2C55 from 62 (11) to 111 (4) for PPAR $\alpha(p=0.001)$.

Conclusion The results suggest that a functional peroxisome proliferator response element (PPRE) exists within the promoter regions of CYP2C40 and CYP2C55 and that activation of PPAR $\alpha$ within the HCA-7 cell line leads to a significant increase in CYP2C40 and CYP2C55 expression. Given the beneficial properties of PPAR $\alpha$ and CYP derived AA metabolites it seems that CYP2C40 and CYP2C55 may become important future therapeutic targets in colon cancer.

Competing interests None declared. 